\title{
Author Correction: A deep-learning pipeline for the diagnosis and discrimination of viral, non-viral and COVID-19 pneumonia from chest X-ray images
}

Guangyu Wang (D), Xiaohong Liu, Jun Shen (D), Chengdi Wang, Zhihuan Li, Linsen Ye, Xingwang Wu, Ting Chen (iD, Kai Wang, Xuan Zhang, Zhongguo Zhou, Jian Yang, Ye Sang, Ruiyun Deng, Wenhua Liang, Tao Yu, Ming Gao, Jin Wang, Zehong Yang, Huimin Cai, Guangming LuDiD, Lingyan Zhang, Lei Yang, Wenqin Xu, Winston Wang (D), Andrea Olvera, Ian Ziyar, Charlotte Zhang, Oulan Li, Weihua Liao, Jun Liu BD, Wen Chen, Wei Chen, Jichan Shi, Lianghong Zheng, Longjiang Zhang, Zhihan Yan, Xiaoguang Zou, Guiping Lin, Guiqun Cao, Laurance L. Lau, Long Mo DD, Yong Liang, Michael Roberts (D), Evis Sala (D), Carola-Bibiane Schönlieb, Manson Fok, Johnson Yiu-Nam Lau, Tao Xu, Jianxing He, Kang Zhang (D), Weimin Li and Tianxin Lin (D)

Correction to: Nature Biomedical Engineering https://doi.org/10.1038/s41551-021-00704-1, published online 15 April 2021.

In the version of this Article originally published, the surname of the author Andrea Olvera was mistakenly spelt as 'Olevera'; it has now been corrected in all versions of the Article.

Published online: 29 July 2021

https://doi.org/10.1038/s41551-021-00787-w

(C) The Author(s), under exclusive licence to Springer Nature Limited 2021 